\title{
IIMIII Building Equity Improvement into Quality Improvement: Reducing Socioeconomic Disparities in Colorectal Cancer Screening as Part of Population Health Management
}

\author{
Seth A. Berkowitz, MD MPH',2, Sanja Percac-Lima, MD PhD ${ }^{1,2}$, Jeffrey M. Ashburner, , MPH', \\ Yuchiao Chang, PhD ${ }^{1,2}$, Adrian H. Zai, MD PhD MPH ${ }^{2,3}$, Wei He, MS ${ }^{7}$, Richard W. Grant, MD MPH', \\ and Steven J. Atlas, MD MPH',
}

\begin{abstract}
'Division of General Internal Medicine, Medical Services, Massachusetts General Hospital, Boston, MA, USA; ${ }^{2}$ Harvard Medical School, Boston, MA, USA; ${ }^{3}$ Laboratory of Computer Science, Massachusetts General Hospital, Boston, MA, USA; ${ }^{4}$ Division of Research, Kaiser Permanente Northern California, Oakland, CA, USA.
\end{abstract}

BACKGROUND: Improving colorectal cancer (CRC) screening rates for patients from socioeconomically disadvantaged backgrounds is a recognized public health priority.

OBJECTIVE: Our aim was to determine if implementation of a system-wide screening intervention could reduce disparities in the setting of improved overall screening rates. DESIGN: This was an interrupted time series (ITS) analysis before and after a population management intervention.

PARTICIPANTS: Patients eligible for CRC screening (age 52-75 years without prior total colectomy) in an 18practice research network from 15 June 2009 to 15 June 2012 participated in the study.

INTERVENTION: The Technology for Optimizing Population Care (TopCare) intervention electronically identified patients overdue for screening and facilitated contact by letter or telephone scheduler, with or without physician involvement. Patients identified by algorithm as high risk for non-completion entered into intensive patient navigation.

MAIN MEASURES: Patients were dichotomized as $\leq$ high school diploma ( $\leq \mathrm{HS}$ ), an indicator of socioeconomic disadvantage, vs. $>$ HS diploma ( $>$ HS). The monthly disparity between $\leq \mathrm{HS}$ and $>\mathrm{HS}$ with regard to $\mathrm{CRC}$ screening completion was examined.

KEY RESULTS: At baseline, $72 \%$ of 47,447 eligible patients had completed screening, compared with $75 \%$ of 51,442 eligible patients at the end of follow-up $(p<0.001)$. CRC screening completion was lower in $\leq \mathrm{HS}$ vs. $>\mathrm{HS}$ patients in June 2009 (65.7\% vs. $74.5 \%, p<0.001)$ and remained lower in June 2012 (69.4 \% vs. $76.7 \%$, $p<0.001)$. In the ITS analysis, which accounts for secular trends, TopCare was associated with a significant decrease in the CRC screening disparity $(0.7 \%, p<0.001)$. The effect of TopCare represents approximately 99 additional $\leq$ HS patients screened above prevailing trends, or 26 life-years gained had these patients remained unscreened.

Received August 24, 2014

Revised January 7, 2015

Accepted January 26, 2015

Published online February 13, 2015
CONCLUSIONS: A multifaceted population management intervention sensitive to the needs of vulnerable patients modestly narrowed disparities in CRC screening, while also increasing overall screening rates. Embedding interventions for vulnerable patients within larger population management systems represents an effective approach to increasing overall quality of care while also decreasing disparities.

KEY WORDS: colorectal cancer; cancer screening; health status disparities; socioeconomic factors; quality improvement.

J Gen Intern Med 30(7):942-9

DOI: $10.1007 / \mathrm{s} 11606-015-3227-4$

(C) Society of General Internal Medicine 2015

$\mathrm{C}$ olorectal cancer (CRC), the third leading cause of cancer mortality for women and men in the United States, ${ }^{1,2}$ can be effectively prevented through several screening strategies. ${ }^{3}$ Though colorectal cancer screening rates have improved over the past decade, screening in most healthcare systems remains suboptimal. ${ }^{4}$ In 2010 , approximately $65 \%$ of eligible patients reported being up to date on screening, ${ }^{5}$ and it is estimated that almost 19,000 lives per year could be saved if population CRC screening goals were obtained. ${ }^{6}$ This is particularly true for socioeconomically disadvantaged patients, who are less likely to be screened and more likely to die from $\mathrm{CRC}$. ${ }^{4-9}$ As a result, improving CRC screening for patients with low educational attainment, an indicator of low socioeconomic status, has been identified as a public health priority. ${ }^{4,5}$

Previous studies have demonstrated that several approaches for increasing overall CRC screening and reducing disparities in CRC screening are possible, such as mailed interventions, telephone outreach, and patient navigation. ${ }^{10-21}$ However, it is unclear whether a pragmatic intervention targeting screening in large healthcare delivery systems with diverse practice types can simultaneously achieve these twin aims of quality and equity improvement. Programs targeting population-level screening rates may actually increase disparities if they are 
more successful in well-off groups, ${ }^{13}$ while interventions meant to improve equity may not increase overall screening rates. ${ }^{22}$

We conducted a pragmatic, multifaceted intervention in a large, urban, academic primary care network to improve overall cancer screening rates; the intervention included efforts to target individuals at increased risk for not completing screening. ${ }^{23}$ In this current analysis, we examined whether this targeted approach was able to reduce disparities while overall CRC screening increased.

\section{METHODS}

\section{Study Design}

Technology for Optimizing Patient Care (TopCare) was a visit-independent population health management intervention developed to increase preventive cancer screening in a primary care practice network. We conducted an interrupted time series analysis to determine whether change in colorectal cancer screening completion rate related to this intervention improved disparities related to level of educational attainment. Because TopCare was implemented system-wide in a randomized study comparing two versions of the intervention, there was no contemporaneous control group for comparisons. Interrupted time series (ITS) is a quasi-experimental study design that allows one to determine whether there has been a change in both level and trend for an outcome after an intervention. ${ }^{24-26}$ Unlike pre/post designs, ITS analysis allows one to model changes in the outcome prior to the intervention, and thus account for 'secular trends' that can otherwise confound assessment of the effectiveness. ${ }^{25}$

\section{Setting and Participants}

This study was conducted in 18 primary care practice sites, including four community health centers, within a practicebased research network in and around Boston, MA that shares a common health information technology (IT) infrastructure. A previously validated automated algorithm was used to identify potential study participants who had at least one visit to any clinic in the primary care network in the 3 years preceding the study period, or during the study period itself. ${ }^{27}$ All such patients were included if they were 52 to 75 years of age, ${ }^{3}$ and did not have evidence of prior total colectomy in their electronic health data. The dates of the study period were 2 years prior to the TopCare intervention study (15 June 2009 to 14 June 2011) and the 1-year study period (15 June 2011 to 15 June 2012).

The Partners Human Research Committee approved this study.

\section{Intervention}

The TopCare intervention consisted of both a health IT platform $^{28}$ and a population management workforce (Clinical
Trials \#NCT01372527). It has been described in greater detail elsewhere, ${ }^{23}$ but in brief, consisted of two main elements: 1 ) electronic identification of eligible patients overdue for screening, and 2) workflow to contact them and track test completion. Contact of patients could take several forms. For most patients, an initial reminder letter (in English or Spanish based on patient's preferred language) was mailed, which contained low-literacy-appropriate educational materials regarding CRC screening and contact information to facilitate scheduling a screening test with a "practice delegate." At the time the letter was mailed, the patient was placed on the electronic list of a practice delegate, who monitored whether the overdue patient had completed screening. Delegates could receive calls from patients and/or conduct telephone outreach to help patients with scheduling. If a patient was identified as high-risk for screening non-completion (based on an electronic algorithm using the number of overdue tests, clinic 'no-shows,' primary language other than English, and age) and remained incomplete on the delegate's list after 4 months, the patient was referred to a patient navigator. The patient navigator delivered intensified outreach in English, Spanish, or Portuguese, and utilized other interpreter resources to assist patients speaking one of 18 other languages. This intensified outreach could include individualized counseling and education, teamwork to overcome barriers to screening, arranging transportation, and accompaniment to screening visits. Patients designated by their primary care provider as likely to benefit from enhanced contact could be referred directly to the patient navigator without waiting for the intervening period.

\section{Outcome Measures}

Patient characteristics, including educational attainment, were abstracted from an electronic health data repository. ${ }^{29}$ For this study, in accord with the national health disparities report, ${ }^{4}$ we dichotomized educational attainment as $\leq$ high school diploma, indicating low educational attainment, or $>$ high school diploma, indicating high educational attainment. The primary outcome for this study was the systemlevel difference in CRC screening completion rate by educational attainment.

For each 1-month observation period, we first established the population of patients eligible for CRC screening. This consisted of all patients associated with the network who were eligible based on age and did not meet exclusion criteria. Eligible patients were then considered to have completed screening if there was an electronic report or billing record of the patient having undergone colonoscopy in the past 10 years, or sigmoidoscopy, barium enema, orcomputed tomography $(\mathrm{CT})$ colonography in the past 5 years. During the study period, fecal immunochemical testing (FIT) was not used by any practice. Patients who declined other methods of CRC screening could be screened by home fecal occult blood testing (FOBT). However, because optical screening 
was the network's preferred approach and documentation of FOBT results in the electronic health record was poor, home FOBT was not included in the outcome assessment. The system level disparity in CRC completion was calculated as the difference in the percentage of screened patients among patients eligible, for those with low vs. high educational attainment. To account for changes in patient age, screening eligibility, and network association, we used an open cohort design. Therefore, individual patients contributed data for the calculation of screening completion rates during time periods they were associated with the network and eligible for screening.

\section{Statistical Analysis}

The main TopCare clinical trial tested two different versions of the TopCare system, one that involved the primary care provider (PCP) to facilitate targeted screening outreach, and one that was fully automated, without provider review. Because our intent for this analysis was to evaluate changes in CRC screening disparities by educational attainment after the implementation of TopCare, rather than to compare two different methods of patient contact, and because the effectiveness of both versions of TopCare was similar, ${ }^{23}$ we combined both arms of the trial for this analysis.

Patient characteristics at the start of the TopCare intervention between low and high educational attainment patients were compared using chi-squared and $t$ tests. We compared the screening completion rate between June 2009 and June 2012 using logistic regression models with generalized estimating equations (GEEs) to account for repeated observations within patients.

For the ITS analysis, we compared the CRC screening disparity by educational attainment present in the network on the 15 th day of each month at 24 points before, and 10 points after, the implementation of TopCare. Because the intervention started 15 June 2011, we considered 15 June 2009 to 14 June 2011 to represent the 'before TopCare' period. We considered the assessments from 15 September 2011 to 15 June 2012 to represent the 'after TopCare' period, with the remaining time comprising the 'transition' period. The transition period $^{25,26}$ accounts for an inevitable delay between the start of an intervention and the time a patient could first complete screening due to the intervention, such as time to receive a reminder letter, and then schedule and attend a colonoscopy. To ensure that the length of the transition period we specified did not affect the conclusions of the analysis, we conducted sensitivity analyses that varied the length of the transition period from 1 to 3 months. At each observation point, there were approximately 14,000 low educational attainment, and 35,000 high educational attainment patients eligible for CRC screening. We tested for differences in the intercept, the overall screening disparity, and the slope, the rate of change in the screening disparity, by testing whether the intercept and slope terms from ordinary least squares regression lines fit for data obtained during the 'after TopCare' period had changed from the terms estimated using data obtained from the 'before TopCare' period. In order to account for autocorrelation, we conducted the analysis using PROC AUTOREG in SAS version 9.3 (Cary, NC). Because one community health center (CHC) practice, the Chelsea HealthCare Center, already had a robust CRC screening program that pioneered the use of patient navigation for cancer screening in our system, ${ }^{15,16}$ which was then expanded to the other practices in the TopCare intervention, we conducted stratified analyses where we separately examined this $\mathrm{CHC}$ and all other practices. Other than this $\mathrm{CHC}$, no practices had any elements of the TopCare program prior to its implementation, and no other programs to improve CRC screening were implemented in our system contemporaneous with TopCare.

In addition to the system-level ITS analysis, we also conducted several patient-level descriptive and exploratory analyses using chi-squared and t-tests. When analyzing patient-level data over time, we used logistic regression with generalized estimating equations (PROC GENMOD) to account for repeated observations within patients.

\section{RESULTS}

At the start of the TopCare intervention (June 2011), there were 49,733 patients in the practice network eligible for CRC screening, of whom $56 \%$ were women and the mean age was 62 years (Table 1). Compared with high educational attainment patients, low education attainment patients were more likely to self-identify as a racial/ethnic minority, have Medicaid insurance, and have a primary language other than English.

Measured results revealed significant changes in CRC screening disparities during the time period. On 15 June 2009, 34,140 of 47,447 (72.0\%) eligible patients had completed CRC screening. Patients with low, compared with high, educational attainment had lower CRC screening completion rates (65.7 \% vs. $74.5 \%, p<0.001)$. On 15 June 2012, 38,402 of 51,442 (74.7\%) eligible patients had completed CRC screening, with lower rates among those with low, compared with high, educational attainment $(69.4 \%$ vs. $76.7 \%, p<0.001)$. Comparing these two time points with repeated measures GEE models, there was a significant increase in CRC screening completion overall (2.7\% increase, $p<0.001)$ and for both low (3.7\% increase, $p<0.001)$, and high $(2.2 \%$ increase, $p<0.001)$ educational attainment patients.

In estimates derived from the ITS analyses (Fig. 1 and Table 2), CRC disparities by educational attainment declined prior to TopCare $(0.04 \%$ decrease per month, $p=0.002)$ (Table 2). Even accounting for this, the TopCare intervention reduced the CRC screening disparity $(0.68 \%$ decrease, $p<0.001$ ), with no change in the improving trend postTopCare (trend change $0.001 \%, p=0.48$ ). In absolute terms, narrowing the screening disparity for 14,693 patients with low educational attainment represents approximately 99 low educational attainment patients screened in addition to the main 
Table 1 Patient Characteristics at Start of TopCare Intervention

\begin{tabular}{|c|c|c|c|c|}
\hline & Overall & $\leq \mathbf{H S}$ & $>$ HS & $p$ \\
\hline & $\begin{array}{l}\% \text { or mean(sd) } \\
N=49,733\end{array}$ & $\begin{array}{l}\% \text { or mean(sd) } \\
N=14,693\end{array}$ & $\begin{array}{l}\text { \% or mean(sd) } \\
N=35,040\end{array}$ & \\
\hline Age, years & $61.9(6.5)$ & $62.5(6.8)$ & $61.7(6.4)$ & $<0.001$ \\
\hline Women & 55.5 & 56.6 & 55.0 & 0.001 \\
\hline Race/Ethnicity & & & & $<0.001$ \\
\hline Non-Hispanic White & 83.3 & 68.5 & 89.5 & \\
\hline Non-Hispanic Black & 5.6 & 9.0 & 4.2 & \\
\hline Hispanic & 6.1 & 15.0 & 2.3 & \\
\hline Asian/ other & 4.8 & 7.4 & 3.8 & \\
\hline Insurance & & & & $<0.001$ \\
\hline Commercial & 62.0 & 47.5 & 68.1 & \\
\hline Medicare & 27.6 & 36.6 & 23.8 & \\
\hline Medicaid & 7.9 & 12.8 & 5.8 & \\
\hline Free-care & 0.2 & 0.3 & 0.1 & \\
\hline Self-pay & 2.5 & 2.9 & 2.3 & \\
\hline Primary language other than English & 8.5 & 33.0 & 2.4 & $<0.001$ \\
\hline Census tract median household income, $\$$ & $64,324(31,717)$ & $49,076(22,114)$ & $70,871(32,943)$ & $<0.001$ \\
\hline Primary care practice is community health center & 22.8 & 45.5 & 13.3 & $<0.001$ \\
\hline
\end{tabular}

$\leq$ HShigh school diploma or less educational attainment; > HSsome college or higher educational attainment

effect of the intervention on screening, or a change that would have taken 17 months to occur without TopCare. Because Fig. 1 gave the visual appearance of having two distinct periods prior to TopCare, a relatively constant initial 12-month period followed by a down-trending period, we conducted a sensitivity analysis to determine if analyzing these as distinct periods, rather than combining them, would affect the estimate of TopCare's effect. The results of this sensitivity analysis are similar to the main analysis $(0.74 \%$ decrease due to TopCare, $p=0.002)$. Additionally, sensitivity analyses that varied the time of the 'transition' period yielded similar results to the main analysis (data available upon request).
In analyses (Table 2 and Fig. 2) examining the practices (all but one in the network) that did not have a CRC screening program prior to TopCare, there was no evidence of improvement in CRC screening disparities by educational attainment prior to TopCare $(0.01 \%$ improvement/month, $p=0.20)$, but the introduction of TopCare significantly reduced disparities (0.69\% improvement, $p<0.001)$. In the one CHC with a CRC screening program prior to TopCare, there was significant improvement over the entire study period $(0.18 \%$ improvement/month, $p<0.001$ ), with elimination of the disparity by the end of the study period (CRC screening completion $71.5 \%$ in low educational attainment group vs. $71.8 \%$ in high educational attainment, $p=0.83$ ). The introduction of

Differences in CRC Screening Completion by Educational Attainment

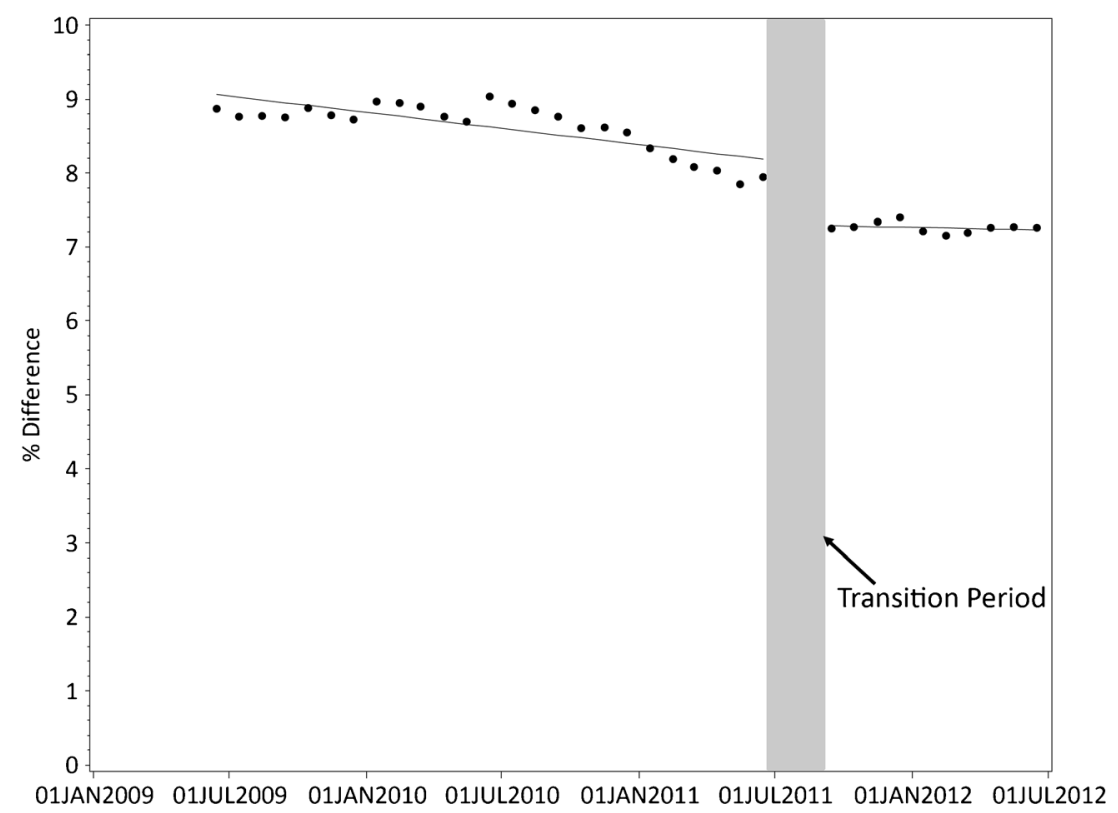

Figure 1 Differences in CRC screening by educational attainment. Dots represent actual measured colorectal cancer screening rates at each time point. A line plotting the regression equation derived from the interrupted time series analysis is superimposed. 
Table 2 Interrupted Time Series Model Estimates for Difference in Colorectal Cancer Screening Completion by Educational Attainment

\begin{tabular}{lcc}
\hline \hline & \% Difference* & $p$ \\
\hline Primary analysis: All clinics & & \\
Baseline level & 9.6 & $<0.001$ \\
Trend prior to TopCare & -0.04 & 0.002 \\
TopCare effect & -0.68 & $<0.001$ \\
Change in trend after TopCare & 0.0008 & 0.48 \\
Stratified analysis: All clinics except community health center & \\
with pre-existing patient navigation & 8.93 & $<0.001$ \\
Baseline level & -0.01 & 0.20 \\
Trend prior to TopCare & -0.69 & $<0.001$ \\
TopCare effect & 0.0003 & 0.77 \\
Change in trend after TopCare & & \\
Stratified analysis: Community health center with pre-existing & \\
patient navigation & 4.99 & $<0.001$ \\
Baseline level & -0.18 & $<0.001$ \\
Trend prior to TopCare & 0.16 & 0.74 \\
TopCare effect & 0.004 & 0.08 \\
Change in trend after TopCare & & \\
\hline
\end{tabular}

* Model estimate represents difference in \% of eligible patients who completed colorectal cancer screening in high minus low educational attainment group (negative values represent decreasing disparity)

All models also account for autocorrelation

TopCare, which contained many elements already present in one CHC's program, was not associated with a significant reduction in CRC screening disparities by educational attainment at that $\mathrm{CHC}(0.16 \%$ change, $p=0.74)$.

\section{DISCUSSION}

In this pragmatic intervention to improve preventive cancer screening within a primary care practice network, disparities in colorectal cancer screening by educational attainment modestly decreased, while overall screening completion in the population increased. Over the study period, CRC screening increased in both high and low educational attainment groups, with moderately more screening in the low educational attainment group attributable to the TopCare intervention. To put this improvement in perspective, bringing the additional low educational attainment patients screened using TopCare into an every-10-year-with-colonoscopy-from-age-50-to-75 screening strategy represents approximately 26 life-years gained, compared with those patients not being screened, based on the Simulation Model of Colorectal Cancer (SimCRC). ${ }^{3}$ While there was an overall trend towards improvement in CRC screening disparities by educational attainment prior to TopCare, the stratified analyses demonstrate that this was related to CRC screening efforts at one community health center. Practices that had no concerted efforts to improve CRC screening beyond standard visit-based reminders did not show significant improvement prior to TopCare. While the absolute reduction in disparities is small, given that national CRC screening disparities actually increased from 2000 to 2010 in vulnerable patients, ${ }^{30}$ and the potential for interventions to increase disparities (even while increasing overall screening), ${ }^{13}$ we believe the reduction in disparities in this study may be important from a population health perspective, especially in the setting of overall screening improvement.

This study is consistent with and expands previous literature. Prior studies have shown that clinical interventions can improve overall CRC screening, ${ }^{10,13,17,31}$ and be effective in vulnerable populations. ${ }^{11,12,14,15,18,20,21,32}$ A recent study from our research group showed that culturally tailored patient

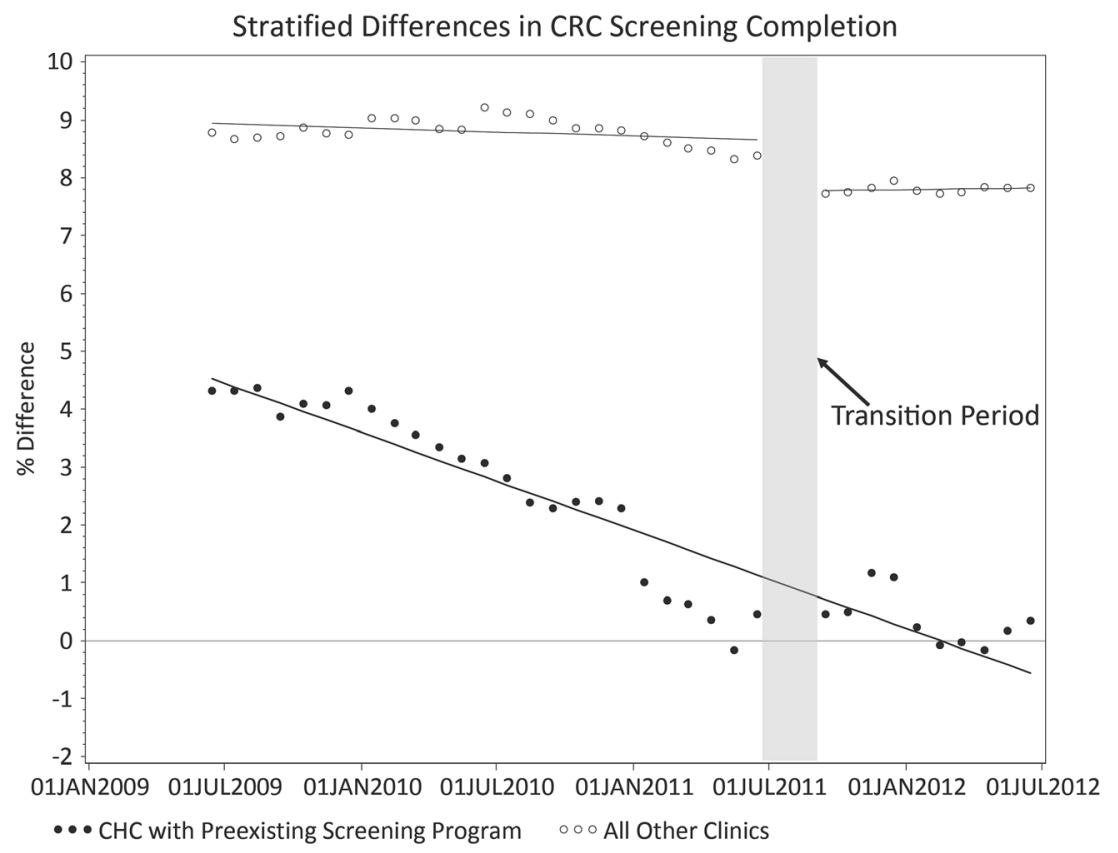

Figure 2 Differences in CRC Screening by educational attainment, stratified by presence of CRC screening program in clinic prior to TopCare. Dots/circles represent actual measured colorectal cancer screening rates at each time point. A line plotting the regression equation derived from the interrupted time series analysis is superimposed. 
navigation for $\mathrm{CRC}$ screening in a community health center eliminated disparities in CRC screening in Latinos and nonEnglish speakers in the health center, but paradoxically created a disparity in vulnerable patients receiving care in other practices within the primary care network. ${ }^{16}$ In the current study, by integrating both health IT and a system-wide population management workforce, we demonstrated that it is possible to improve both quality and equity at the same time in a large healthcare delivery system. We believe that this is important, because overall, it is not yet known whether population health management approaches will increase, decrease, or not affect disparities in care. As population health management strategies increasingly become part of routine care, being able to address disparities in this way may help move disparity measurement from a number calculated largely for research purposes to an important indicator of overall system functioning that can be targeted for operations improvement. Part of this intervention's success may lie in the ability to target interventions more efficiently - utilizing 'low touch' techniques for the majority of patients, while directing 'high-touch,' resource-intensive components to those most likely to benefit. The use of health IT to accomplish this targeting allowed for expansion of the highly effective patient navigation from a single clinic to a system-wide program.

This study has several implications for healthcare delivery and population management. Quality improvement interventions often include multiple components targeting several different aspects of care. This provides an opportunity to address both quality and equity in the same intervention. Strategies such as using educational materials appropriate for patients with low literacy, recognizing heterogeneity in socioeconomic circumstances and barriers to care, and allowing for more intensive patient education and counseling in participants at high-risk for poor outcomes are broadly applicable to population management programs. By consciously pursuing both a quality and equity agenda during intervention design, the false choice between improving quality without attention to improving equity can be avoided. ${ }^{33}$

While the proportion of low educational attainment patients was higher at the one community health center with an existing navigator program (as well as at other network community health centers) than the rest of the practices in the network, the majority of low educational attainment patients in the network received care at practices other than the health centers. In this model, an academic community health center served as a 'lab' to produce an intervention for equity improvement that was then deployed in other practices. Using community health centers to 'incubate' programs for practices with fewer vulnerable patients and less incentive to design equity improvement interventions may be a broadly applicable approach to reducing disparities in diverse healthcare systems, and shows that including community health centers in academic healthcare delivery systems can advance the research mission alongside the educational and clinical missions.
The results of this study should be interpreted in the context of several limitations. Because TopCare was implemented as a new standard of care throughout the healthcare system, there was no contemporaneous control group. However, by using the ITS design, we can have confidence that 'secular trends' were not solely responsible for the improvement in CRC screening disparities we observed. Next, the TopCare system was studied in a single healthcare delivery network with an advanced IT infrastructure. However, the network does include a diversity of practice types, including urban and suburban clinics, teaching and non-teaching practices, as well as community health centers and private practices. Additionally, while FOBT testing was offered as a screening option, we were unable to count as complete patients screened in this way. Because patients with low educational attainment may preferentially choose home FOBT screening over colonoscopy,${ }^{34}$ we may have underestimated the reduction in CRC screening disparities attributable to TopCare by not counting the FOBT testing that was done. Additionally, by de-emphasizing what may have been a preferred strategy in this group, it may have served as a barrier to further reductions. ${ }^{19}$ Finally, Massachusetts has a very high rate of CRC screening. While the national average CRC screening rate for all patients was $65 \%$ in $2010,{ }^{5}$ at the beginning of this study, in 2009, CRC screening rates in our system were already $72 \%$ overall and $66 \%$ for low educational attainment patients. Thus, there may have been a 'ceiling' effect with regard to increasing CRC screening. The application of a TopCare-like approach in systems with lower initial rates of CRC screening has the potential to provide even greater benefits than observed here.

These limitations are balanced by several strengths. Since virtually all included patients had coverage for CRC screening, including colonoscopy, issues of access are less of a concern, providing a 'cleaner' test of the intervention itself. Also, the use of a validated 'linkage' algorithm to identify patients with ongoing care within our network increases confidence that patients were not receiving CRC screening in outof-network settings, ${ }^{27}$ and the quasi-experimental design overcomes limitations of patient selection and generalizability noted in previous studies. ${ }^{13,25}$

While educational disparities in CRC screening were reduced by the TopCare intervention, they were not eliminated. Further, while the overall disparity was reduced, the lack of improvement in the rate of change in the disparity speaks to the limits of the current intervention. To accelerate resolution of screening disparities, elements of the program that are particularly effective for disparity reduction may need to be expanded, or additional elements may need to be added. The analytic methods we used do not permit determination of which TopCare elements were most effective. However, visual inspection of the data does suggest decreases in disparities relatively soon after implementation, before many patients would have received navigation. Given prior work suggesting vulnerable patients may be less likely to have had a recommendation for screening, ${ }^{35,36}$ and the success of mailings in 
other studies, ${ }^{10,13,17,31,37}$ it may have been that there were differentially larger numbers of lower education patients who had had little prompting regarding screening prior to TopCare. We do know, however, that patient navigation is very effective for the patients navigated. ${ }^{16}$ This intervention focused on the healthcare delivery system, and so we have little data on patient reasons for non-completion, which may include less education regarding the importance and benefits of screening, barriers to screening such as transportation, obtaining time off of work, and lost wages, and differences in psychological factors such as time-orientation. ${ }^{32,38,39}$ To make greater improvements in equity than were achieved with this intervention, future research may need to identify patients with these barriers, and target interventions to overcome them. Inclusion of education or other socioeconomic data in identification algorithms may be particularly useful. ${ }^{20}$ To be able to eliminate disparities in cancer prevention, the programs implemented should be sustainable, continuous, and incorporated as a part of usual care. ${ }^{16}$

In conclusion, a multifaceted intervention that combined health IT and a population management workforce successfully reduced CRC screening disparities by educational attainment in the setting of improved overall screening. Those designing quality improvement interventions may wish to consider building in components focused on decreasing disparities and measure their intervention's impact on both care quality and equity.

Acknowledgements: Funding: This study was supported by grants from the Agency for Healthcare Research and Quality (AHRQ R18HSO18161), the Controlled Risk Insurance Company/Risk Management Foundation, and institutional funding through the Massachusetts General Hospital Primary Care Operations Improvement Program and the Massachusetts General Physicians Organization. Seth A. Berkowitz was supported by an Institutional National Research Service Award \#T32HP10251, the Ryoichi Sasakawa Fellowship Fund, and by the Division of General Internal Medicine at Massachusetts General Hospital.

Prior Presentation: An earlier version of this manuscript was presented at the New England Regional SGIM meeting, 7 March 2014, in Boston, MA, and at the National SGIM meeting, 25 April 2014, in San Diego, CA.

Conflict of Interest: Massachusetts General Hospital entered into a royalty arrangement on 27 June 2013 , to commercialize the population management system with SRG Technology, a for-profit company. AHZ and SJA are beneficiaries of this royalty arrangement, but have not received any payments to date. In addition, $A H Z$ is a part-time employee of SRG Technology. All other authors report no conflicts of interest.

Corresponding Author: Seth A. Berkowitz, MD MPH; Harvard Medical School, Boston, MA, USA (e-mail: SABerkowitz@partners.org).

\section{REFERENCES}

1. Siegel R, Desantis C, Jemal A. Colorectal cancer statistics, 2014. CA Cancer J Clin. 2014;64(2):104-17. doi:10.3322/caac.21220.

2. U.S. Cancer Statistics Working Group. United States Cancer Statistics: 1999-2010 Incidence and Mortality Web-based Report. . U.S. Department of Health and Human Services, Centers for Disease Control and Prevention and National Cancer Institute, Atlanta. 2013. www.cdc.gov/uscs. Accessed 28 January 2015.

3. U.S. Preventive Services Task Force. Screening for Colorectal Cancer: U.S. Preventive Services Task Force Recommendation Statement. AHRQ Publication 08-05124-EF-3 2008. http://www.uspreventiveservicestaskforce.org/ uspstf08/colocancer/colors.htm. Accessed 28 January 2015.

4. Agency for Healthcare Research and Quality. National Healthcare Quality \& Disparities Reports. . Rockville, MD. 2014. http://www.ahrq.gov/ research/findings/nhqrdr/index.html. Accessed 28 January 2015.

5. Steele CB, Rim SH, Joseph DA, King JB, Seeff LC. Colorectal cancer incidence and screening - United States, 2008 and 2010. MMWR Surveill Summ. 2013;62(3):53-60.

6. Maciosek MV, Solberg LI, Coffield AB, Edwards NM, Goodman MJ. Colorectal cancer screening: health impact and cost effectiveness. Am J Prev Med. 2006;31(1):80-9. doi:10.1016/j.amepre.2006.03.009.

7. Centers for Disease Control and Prevention. Vital signs: colorectal cancer screening test use-United States, 2012. MMWR Morb Mortal Wkly Rep. 2013;62(44):881-8.

8. Saldana-Ruiz N, Clouston SA, Rubin MS, Colen CG, Link BG. Fundamental causes of colorectal cancer mortality in the United States: understanding the importance of socioeconomic status in creating inequality in mortality. Am J Public Health. 2013;103(1):99-104. doi:10. 2105/AJPH.2012.300743.

9. Liss DT, Baker DW. Understanding current racial/ethnic disparities in colorectal cancer screening in the United States: the contribution of socioeconomic status and access to care. Am J Prev Med. 2014;46(3):228-36. doi:10.1016/j.amepre.2013.10.023.

10. Baker DW, Brown T, Buchanan DR, Weil J, Balsley $\mathbf{K}$, Ranalli L, et al. Comparative effectiveness of a multifaceted intervention to improve adherence to annual colorectal cancer screening in community health centers: a randomized clinical trial. JAMA Intern Med. 2014. doi:10.1001/ jamainternmed.2014.2352.

11. Dietrich AJ, Tobin JN, Cassells A, Robinson CM, Greene MA, Sox CH, et al. Telephone care management to improve cancer screening among lowincome women: a randomized, controlled trial. Ann Intern Med. 2006; 144(8):563-71.

12. Dietrich AJ, Tobin JN, Robinson CM, Cassells A, Greene MA, Dunn VH, et al. Telephone outreach to increase colon cancer screening in medicaid managed care organizations: a randomized controlled trial. Ann Fam Med. 2013;11(4):335-43. doi:10.1370/afm. 1469.

13. Green BB, Wang CY, Anderson ML, Chubak J, Meenan RT, Vernon SW, et al. An automated intervention with stepped increases in support to increase uptake of colorectal cancer screening: a randomized trial. Ann Intern Med. 2013;158(5 Pt 1):301-11. doi:10.7326/0003-4819-158-5201303050-00002.

14. Lasser KE, Murillo J, Lisboa S, Casimir AN, Valley-Shah L, Emmons $\mathbf{K M}$, et al. Colorectal cancer screening among ethnically diverse, lowincome patients: a randomized controlled trial. Arch Intern Med. 2011;171(10):906-12. doi:10.1001/archinternmed.2011.201.

15. Percac-Lima S, Grant RW, Green AR, Ashburner JM, Gamba G, Oo S, et al. A culturally tailored navigator program for colorectal cancer screening in a community health center: a randomized, controlled trial. J Gen Intern Med. 2009;24(2):211-7. doi:10.1007/s11606-008-0864-x.

16. Percac-Lima S, Lopez L, Ashburner JM, Green AR, Atlas SJ. The longitudinal impact of patient navigation on equity in colorectal cancer screening in a large primary care network. Cancer. 2014;120(13):2025-31. doi: $10.1002 /$ cncr.28682.

17. Sequist TD, Zaslavsky AM, Marshall R, Fletcher RH, Ayanian JZ. Patient and physician reminders to promote colorectal cancer screening: a randomized controlled trial. Arch Intern Med. 2009;169(4):364-71. doi:10. 1001/archinternmed.2008.564.

18. Carney PA, Lee-Lin F, Mongoue-Tchokote S, Mori M, Leung H, Lau C, et al. Improving colorectal cancer screening in Asian Americans: results of a randomized intervention study. Cancer. 2014;120(11):1702-12. doi:10. 1002/cncr.28640.

19. Gupta S, Sussman DA, Doubeni CA, Anderson DS, Day L, Deshpande AR, et al. Challenges and possible solutions to colorectal cancer screening for the underserved. J Natl Cancer Inst. 2014;106(4):dju032. doi:10.1093/jnci/dju032

20. Jandorf L, Braschi C, Ernstoff E, Wong CR, Thelemaque L, Winkel G, et al. Culturally targeted patient navigation for increasing African Americans' adherence to screening colonoscopy: a randomized clinical trial. Cancer Epidemiol Biomarkers Prev. 2013;22(9):1577-87. doi:10. 1158/1055-9965.EPI-12-1275.

21. Sarfaty M, Doroshenk M, Hotz J, Brooks D, Hayashi S, Davis TC, et al. Strategies for expanding colorectal cancer screening at community health centers. CA Cancer J Clin. 2013. doi:10.1002/caac.21191. 
22. Friedman LC, Everett TE, Peterson L, Ogbonnaya KI, Mendizabal V. Compliance with fecal occult blood test screening among low-income medical outpatients: a randomized controlled trial using a videotaped intervention. J Cancer Educ. 2001;16(2):85-8. doi:10.1080/ 08858190109528738.

23. Atlas SJ, Zai AH, Ashburner JM, Chang Y, Percac-Lima S, Levy DE, et al. Non-visit-based cancer screening using a novel population management system. J Am Board Fam Med. 2014;27(4):474-85. doi:10.3122/ jabfm.2014.04.130319.

24. Matowe LK, Leister CA, Crivera C, Korth-Bradley JM. Interrupted time series analysis in clinical research. Ann Pharmacother. 2003;37(7-8):11106.

25. Wagner AK, Soumerai SB, Zhang F, Ross-Degnan D. Segmented regression analysis of interrupted time series studies in medication use research. J Clin Pharm Ther. 2002;27(4):299-309.

26. Zhang F, Wagner AK, Soumerai SB, Ross-Degnan D. Methods for estimating confidence intervals in interrupted time series analyses of health interventions. J Clin Epidemiol. 2009;62(2):143-8. doi:10.1016/j. jclinepi.2008.08.007.

27. Atlas SJ, Grant RW, Ferris TG, Chang Y, Barry MJ. Patient-physician connectedness and quality of primary care. Ann Intern Med. 2009; 150(5):325-35.

28. SRG Technology. TopCare patient population management software. SRG Tech, Inc., Fort Lauderdale, FL. 2014. http://www.srgtech.com/topcare. Accessed 28 January 2015

29. Murphy SN, Chueh HC. A security architecture for query tools used to access large biomedical databases. Proc AMIA Symp. 2002:552-6.

30. Agency for Healthcare Research and Quality. 2012 National Healthcare Disparities Report. In: U. S. Department of Health and Human Services, editor. Rockville, MD2013.

31. Myers RE, Sifri R, Hyslop T, Rosenthal M, Vernon SW, Cocroft J, et al. A randomized controlled trial of the impact of targeted and tailored interventions on colorectal cancer screening. Cancer. 2007;110(9):208391. doi: $10.1002 /$ cncr.23022.

32. Naylor K, Ward J, Polite BN. Interventions to improve care related to colorectal cancer among racial and ethnic minorities: a systematic review. J Gen Intern Med. 2012;27(8):1033-46. doi:10.1007/s11606-012-2044-2.

33. Chin MH, Clarke AR, Nocon RS, Casey AA, Goddu AP, Keesecker NM, et al. A roadmap and best practices for organizations to reduce racial and ethnic disparities in health care. J Gen Intern Med. 2012;27(8):992-1000. doi: 10.1007/s11606-012-2082-9.

34. DeBourcy AC, Lichtenberger S, Felton S, Butterfield KT, Ahnen DJ, Denberg TD. Community-based preferences for stool cards versus colonoscopy in colorectal cancer screening. J Gen Intern Med. 2008;23(2):16974. doi:10.1007/s11606-007-0480-1.

35. Coughlin SS, Thompson T. Physician recommendation for colorectal cancer screening by race, ethnicity, and health insurance status among men and women in the United States, 2000. Health Promot Pract. 2005;6(4):369-78. doi:10.1177/1524839905278742.

36. Wee CC, McCarthy EP, Phillips RS. Factors associated with colon cancer screening: the role of patient factors and physician counseling. Prev Med. 2005;41(1):23-9. doi:10.1016/j.ypmed.2004.11.004.

37. Denberg TD, Coombes JM, Byers TE, Marcus AC, Feinberg LE, Steiner JF, et al. Effect of a mailed brochure on appointment-keeping for screening colonoscopy: a randomized trial. Ann Intern Med. 2006;145(12):895-900.

38. Green AR, Peters-Lewis A, Percac-Lima S, Betancourt JR, Richter JM Janairo MP, et al. Barriers to screening colonoscopy for low-income Latino and white patients in an urban community health center. J Gen Intern Med. 2008;23(6):834-40. doi:10.1007/s11606-008-0572-6.

39. Whitaker KL, Good A, Miles A, Robb K, Wardle J, von Wagner C. Socioeconomic inequalities in colorectal cancer screening uptake: does time perspective play a role? Health Psychol. 2011;30(6):702-9. doi:10. $1037 / \mathrm{a} 0023941$ 